\title{
Asymmetric Charge Partitioning upon Dissociation of DNA Duplexes
}

\author{
James A. Madsen and Jennifer S. Brodbelt \\ Department of Chemistry and Biochemistry, The University of Texas at Austin, Austin, Texas, USA
}

\begin{abstract}
Upon collisional activation, a series of DNA duplexes exhibited a significant degree of asymmetric dissociation with respect to charge partitioning among the single strands. That is, the charge states of the single strand product ions did not equal $\mathrm{q} / 2$ for even precursor charge states or $(q+1) / 2$ and $(q-1) / 2$ for odd precursor charge states (where $q$ is the charge of the precursor). The factors that affect this asymmetric charge partitioning were assessed. The smaller, lower charged duplexes resulted in more symmetric dissociation compared with larger duplexes in higher charge states, which displayed a high degree of asymmetry upon dissociation. The composition of the duplexes influenced charge partitioning, with those containing a greater number of $\mathrm{A} / \mathrm{T}$ base pairs showing more symmetric dissociation relative to the more G/C rich duplexes. The use of higher collisional energies resulted in significantly more asymmetric dissociation. Comparisons were made with the dissociation behavior previously studied for protein noncovalent complexes and past studies of the gas-phase conformations and dissociation of DNA complexes. (J Am Soc Mass Spectrom 2010, 21, 1144-1150) @ 2010 American Society for Mass Spectrometry
\end{abstract}

$\mathrm{T}$ Tandem mass spectrometry, which has proven to be successful for sequencing biopolymers, such as proteins, carbohydrates, and nucleic acids, has been increasingly used to characterize a variety of noncovalent complexes and macromolecules [1, 2]. Activation of noncovalent complexes frequently ruptures the weak noncovalent interactions, causing disassembly into the constituent molecules rather than cleavage of covalent bonds. Numerous recent studies of the dissociation of noncovalent complexes have reported interesting and unexpected asymmetric charge partitioning of the resulting product ions [3-16]. That is, during dissociation one of the monomeric components becomes significantly more enriched in charge compared with other identically massive or even more massive constituents [3-16], and that the charge division is dependent on the surface area of the individual components of the complex [14,17]. This asymmetric charge partitioning has been mainly observed for an array of protein complexes varying both in the size and number of monomers. The generally accepted mechanism for the unusual asymmetric charge partitioning indicates that during activation, certain monomers within the noncovalent complexes become elongated and, thus, have more surface area, which enables them to accept more charges $[9,15]$. A better understanding of this process was made possible by the Thachuk group who modeled protein complex dissociation [17-19]. It was concluded (based on a Coulomb repulsion model) that

Address reprint requests to Dr. J. S. Brodbelt, Department of Chemistry and Biochemistry, The University of Texas at Austin, 1 University Station A5300, Austin TX 78712-0165, USA. E-mail: jbrodbelt@mail.utexas.edu charges should orient themselves uniformly over multimeric complexes, and the symmetrical charge partitioning of the monomers $(\mathrm{q} / 2)$ would afford the lowest energy barrier for dissociation [17]. Jurchen and Williams showed that the origins of asymmetric charge partitioning were dependent on many factors including the precursor charge state and overall flexibility of the intact complex as well as the amount of internal energy added to the complex $[15,16]$. Furthermore, the degree of asymmetric charge partitioning was also shown to depend on the activation method. For example, more symmetric charge partitioning was observed upon surface induced dissociation (SID) compared with collisional induced dissociation (CID) of noncovalent protein complexes [20,21], a result attributed to the more rapid, single step energization process of SID, which limited elongation of the monomers before dissociation of the complexes.

An array of hydrogen bonds provide key stabilization for double stranded DNA, another biopolymer in which noncovalent interactions play a dominant role. Several studies have indicated that many of the solution phase conformations and interactions of DNA are retained in the gas phase. For example, evidence of the preservation of Watson-Crick base pairing interactions in gas-phase duplexes have been shown by strong correlations of solution-phase enthalpies with gas-phase activation energies for dissociation [22, 23], good agreement between gas-phase dissociation and solution-phase thermal denaturing [24], molecular modeling [22, 25], and higher gas-phase stabilities for complementary versus non-complementary duplexes [22]. Gas-phase fluorescence resonance energy transfer 
(FRET) measurements [26, 27] and the observation of terminal base losses [28] have revealed the preferential unzipping of the more weakly bound ends of duplexes upon activation and dissociation. Furthermore, the dissociation of various duplexes in the $7-$ charge state (ones that dissociated symmetrically into single strands retaining three or four charges) were examined by Gabelica and De Pauw to rationalize why certain strands preferentially retained four charges compared to the other complementary strand which retained only three charges [23]. It was found that the identity of the terminal bases was the main factor that governed the uneven charge retention by the two strands, with guanine and thymine bases serving as the most dominant charge sinks [23]. The authors also showed an example of a 16-mer duplex ( $8-$ charge state) that dissociated asymmetrically (producing single strands with five or three charges) [23], but no further examination or explanation was given for this peculiar result. Previous ion mobility studies by Bowers and coworkers have shown that smaller duplexes displayed a more globular structure, while larger duplexes retained their native helical structure in the gas-phase [29-31]. All these previous results motivated our interest in assessing the factors that affect the degree of asymmetric charge partitioning of duplexes upon CID.

In the present study, the charge partitioning of DNA duplexes upon activation and dissociation into the two constituent single strands is examined by CID. We were particularly interested in the production of single strands in which the charges are not split evenly between each strand $(\mathrm{q} / 2$ for precursors in even charge states or $(\mathrm{q}+$ $1) / 2$ and $(q-1) / 2$ for precursors in odd charge states, where $\mathrm{q}$ is the number of charges of the duplex). The degree of asymmetric charge partitioning is compared for a range of charge states of duplexes with varying sizes and base compositions. Dissociation models developed from protein complex dissociation behavior as well as prior knowledge of gas-phase DNA duplex conformations are compared with the results herein.

\section{Experimental}

\section{Materials}

All oligodeoxynucleotides were purchased from Integrated DNA Technologies (Coralville, IA, USA), and all other reagents from Sigma-Aldrich (St. Louis, $\mathrm{MO}$, USA). Single strand concentrations were determined based on Beer's law absorbance measurements at 260 $\mathrm{nm}$ obtained using a spectrophotometer with extinction coefficients provided from Integrated DNA Technologies. Duplexes were formed by annealing two single strands (1 mM each) in $250 \mathrm{mM}$ ammonium acetate after heating at $90^{\circ} \mathrm{C}$ for $10 \mathrm{~min}$, decreasing the temperature by $10^{\circ}$, and subsequently decreasing the temperature every $30 \mathrm{~min}$ by $10^{\circ}$ until room temperature was reached. All DNA duplexes used in this study are listed in Table 1. The nomenclature is as follows: " $\mathrm{d}$ "
Table 1. DNA duplex nomenclature and sequences

\begin{tabular}{|c|c|c|}
\hline $\begin{array}{c}\text { Duplex } \\
\text { name }\end{array}$ & Primary structure & $\begin{array}{l}\text { MW } \\
(\mathrm{Da})\end{array}$ \\
\hline \multirow[t]{2}{*}{ d19-0 } & $5^{\prime}$-CGCGCGCGGCCGCGCGCGG-3' $\left(\mathrm{ss}_{1}\right)$ & 5832.8 \\
\hline & 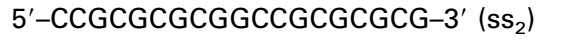 & 5792.7 \\
\hline \multirow[t]{2}{*}{ d19-1 } & 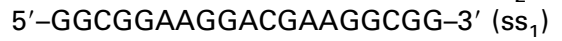 & 5992.9 \\
\hline & 5'-CCGCCTTCGTCCTTCCGCC-3' $\left(\mathrm{ss}_{2}\right)$ & 5627.7 \\
\hline \multirow[t]{2}{*}{ d19-2 } & 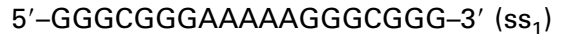 & 6032.9 \\
\hline & $5^{\prime}-\mathrm{CCCGCCCTTTTTCCCGCCC}-3^{\prime}\left(\mathrm{ss}_{2}\right)$ & 5587.6 \\
\hline \multirow[t]{2}{*}{ d19-3 } & $5^{\prime}-$ GGCAAAAAGCGAAAAACGG-3' $\left(\mathrm{ss}_{1}\right)$ & 5912.9 \\
\hline & $5^{\prime}-\mathrm{CCGTTTTTCGCTTTTTGCC-3}\left(\mathrm{ss}_{2}\right)$ & 5702.7 \\
\hline \multirow[t]{2}{*}{ d14-0 } & $5^{\prime}-$ GGCGTCGGCGTCGC-3' $\left(\mathrm{ss}_{1}\right)$ & 4296.8 \\
\hline & $5^{\prime}-$ GCGACGCCGACGCC-3' $\left(\mathrm{ss}_{2}\right)$ & 4234.8 \\
\hline \multirow[t]{2}{*}{ d14-1 } & $5^{\prime}-$ CCGGCGCGCGCCGG-3' $\left(\mathrm{ss}_{1}\right)$ & 4266.8 \\
\hline & 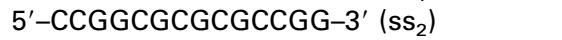 & 4266.8 \\
\hline \multirow[t]{2}{*}{ d14-2 } & 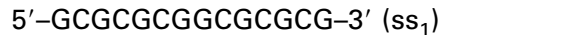 & 4306.8 \\
\hline & $5^{\prime}-$ CGCGCGCCGCGCGC-3' $\left(\mathrm{ss}_{2}\right)$ & 4226.8 \\
\hline \multirow[t]{2}{*}{ d10 } & $5^{\prime}-G C G A A T T G C G-3^{\prime}\left(s_{1}\right)$ & 3068.0 \\
\hline & $5^{\prime}-$ CGCAATTCGC-3' $\left(\mathrm{ss}_{2}\right)$ & 2988.0 \\
\hline \multirow[t]{2}{*}{ d6 } & $5^{\prime}-\mathrm{GCATGG}-3^{\prime}\left(\mathrm{ss}_{1}\right)$ & 1832.3 \\
\hline & $5^{\prime}-\mathrm{CCATGC}-3^{\prime}\left(\mathrm{ss}_{12}\right)$ & 1752.2 \\
\hline
\end{tabular}

stands for duplex, the number following " $\mathrm{d}$ " denotes the number of bases per single strand in the duplex, the number after the hyphen specifies a particular sequence shown in Table 1, and "ss" stands for single strand. Working solutions of $10 \mu \mathrm{M}$ duplex in $50 \mathrm{mM}$ ammonium acetate and $20 \%$ methanol were prepared for direct infusion at a flow rate of $3 \mu \mathrm{L} / \mathrm{min}$. To enhance higher charge states, $0.5 \% \mathrm{~m}$-nitrobenzyl alcohol was spiked into the above working solutions before ESI-MS analysis. Examples of the ESI mass spectra of the duplex d14-0 with and without additions of $m$-NBA can be seen in Supplemental Figure 1, which can be found in the electronic version of this article. Spiking $m$-NBA into the DNA solutions had no impact on the resulting fragmentation patterns of the selected DNA duplexes. For example, CID of the duplex d19-3 in the $8-$ charge state without the addition of $m$-NBA and with $0.5 \%$ m-NBA yielded virtually identical spectra (data not shown).

\section{Mass Spectrometry}

Duplex anions were formed through electrospray ionization and were analyzed using a Thermo Fisher (San Jose, CA, USA) LTQ two-dimensional linear ion trap mass spectrometer. Standard activation times of $30 \mathrm{~ms}$ and a q-value of 0.25 were used for all CID experiments. Ion signals were converted to peak areas using Origin, version 7 (OriginLab, Northampton, MA, USA).

\section{Results and Discussion}

In general, DNA duplexes in lower charge states dissociate via base loss and subsequent dissociation into $\mathrm{a}-\mathrm{B}$ and $\mathrm{w}$ sequence ions (both as single strands and partial duplexes). Significant asymmetric charge distribution is not noted upon dissociation of these lower charged 
duplexes and thus will not be discussed further. In contrast, the product ions resulting from activation of more highly charged duplexes typically dissociate by strand separation with either symmetric or asymmetric charge partitioning between the two resulting strands. Our discussion will focus on these latter species.

Duplexes containing six or 10 base pairs (d6 and d10) yielded charge states ranging from $3-$ to $5-$ and $4-$ to 7-, respectively, upon ESI. The lowest observable charge state of $\mathrm{d} 10$ (4-) dissociated predominantly via formation of $\mathrm{a}-\mathrm{B}$ and $\mathrm{w}$ ions with generally symmetric retention of charges among these product ions. Collisional activation of the 10 base pair duplex in higher charge states (5- to $7-)$ and all charge states (3- to $5-$ ) of the six base pair duplex led predominantly to strand separation with almost exclusive symmetric charge partitioning among the resulting single strands regardless of the initial charge state of the selected precursor ion. For example, d10 in the 6- charge state produced two single strands in 3- charge states, indicating completely uniform charge partitioning among the two constituent strands.

In contrast, the precursor charge state played a significant role on the degree of symmetric versus asymmetric charge partitioning for the larger, more highly charged duplexes. For example, as seen in Figure $1 \mathrm{a}$ and $\mathrm{b}$, the 14 base pair duplex (d14-0) dissociated symmetrically for the lowest charge state precursor ions $(6-, 7-)$, but asymmetric charge partitioning occurred upon dissociation of the 8- precursor ion (Figure 1c). Instead of each strand retaining four charges as would be expected for an even division of charge between the two constituent strands, single strands with three or five charges were more abundant. Figure 2a illustrates the charge partitioning data for the product ions formed upon CID of duplex d14-0 in a format that facilitates

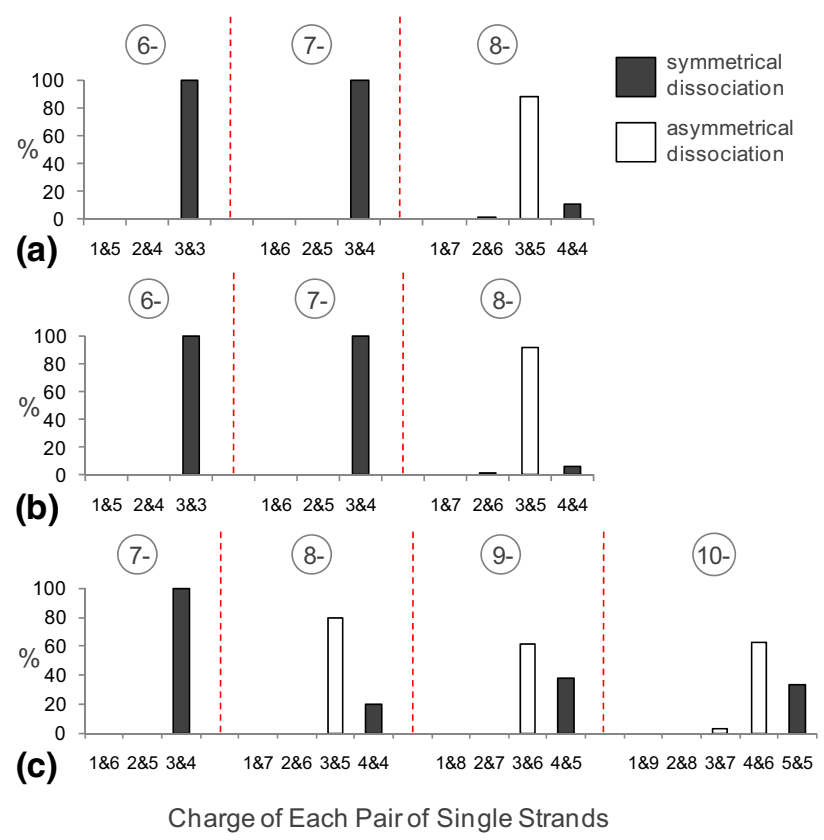

Figure 2. Comparison of symmetrical versus asymmetrical dissociation (a) CID of d14-0, 6- charge state $(47 \mathrm{mV}), 7-$ charge state $(38 \mathrm{mV}), 8$ - charge state $(34 \mathrm{mV})(\mathbf{b})$ CID of d14-2, 6- charge state $(71 \mathrm{mV}), 7-$ charge state $(49 \mathrm{mV}), 8$ - charge state $(45 \mathrm{mV})(\mathrm{c})$ CID of d19-1, 7- charge state $(54 \mathrm{mV}), 8-$ charge state $(44 \mathrm{mV})$, 9 - charge state $(40 \mathrm{mV}), 10$ - charge state $(36 \mathrm{mV})$. The peak areas of the product ions were summed for each pair of single strands and were converted to percentages. The total charge of each pair equaled the precursor charge. The area percentage of product ions involved in charge portioning (asymmetric and symmetric) is plotted against each single strand charge pair.

comparisons between symmetric and asymmetric dissociation. The peak areas of the product ions were summed for each pair of single strands and were converted to percentages. The total charge of each pair
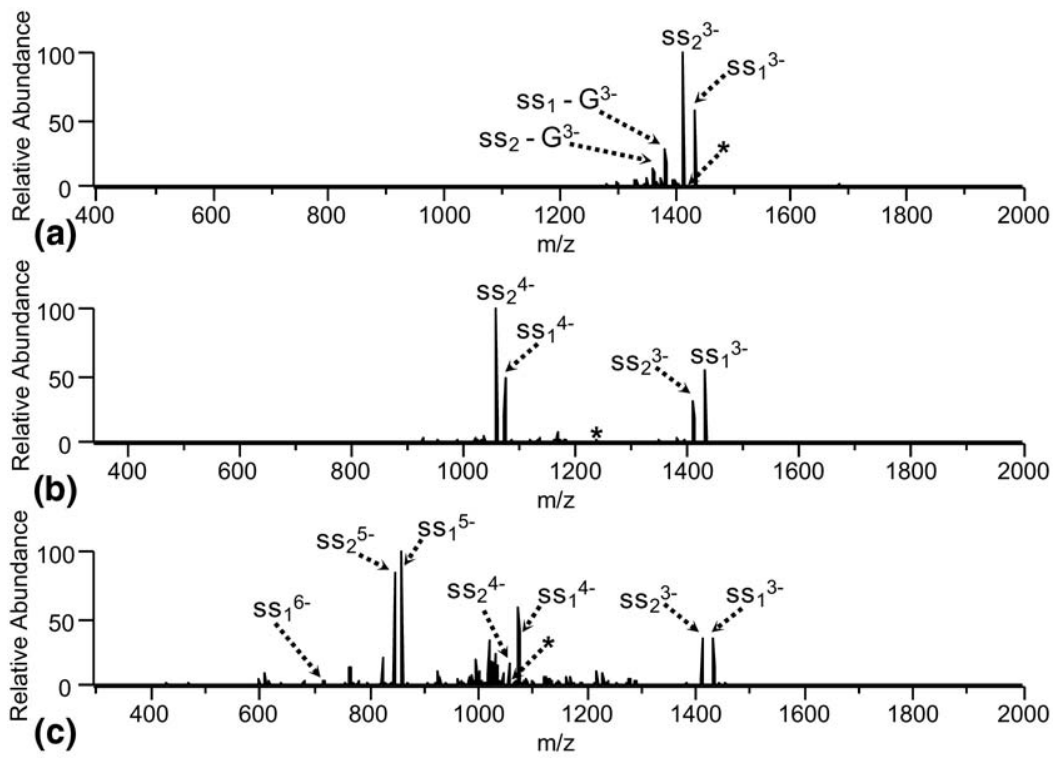

Figure 1. CID of duplex d14-0, (a) 6- charge state, $47 \mathrm{mV}$ (b) 7- charge state, $38 \mathrm{mV}$. (c) 8- charge state, $34 \mathrm{mV}$. The asterisk symbol denotes the precursor ion. 
equaled the precursor charge. The significant asymmetric charge partitioning for the $8-$ precursor is readily apparent in Figure 2a. The dissociation of the selfcomplementary duplex d14-1, which consists of two identical single strands, follows similar trends observed for d14-0; no asymmetric charge partitioning was seen for lower charge states (e.g., 6- and 7-), and significant asymmetric dissociation was observed for higher charge states (e.g., $\geq 8-$ ). Since the $m / z$ values of duplexes in even charge states $(6-, 8-)$ overlap with the $\mathrm{m} / \mathrm{z}$ values of single strand product ions $(3-, 4-)$ for self-complementary duplexes such as d14-1, it is difficult to make systematic comparisons of the asymmetric versus symmetric dissociation behavior for different precursor charge states. Therefore, the duplex d14-2 (Table 1), which is comprised of single strands with nearly uniform base composition (i.e., one strand containing $8 \mathrm{G}$ and $6 \mathrm{C}$, the other containing $6 \mathrm{G}$ and $8 \mathrm{C}$ ), was used instead of d14-1 for charge partitioning comparisons since the $m / z$ values of this duplex do not overlap with any of the single strand products. The same trends observed for d14-0 again hold true for d14-2; lower charge states yielded more symmetrical dissociation and higher charge states produced more asymmetric charge partitioning as seen in Figure 2b. For instance, upon CID of duplex d14-2 in the 8- charge state, over $90 \%$ of the single strand products have either three or five charges with less than $10 \%$ retaining symmetric four charges. Examples of the CID mass spectra of d14-2 (charge states 6- through 8-) are shown in Supplemental Figure 2. The substantial asymmetric charge partitioning that occurs for this nearly self-complementary duplex is striking. Since the strands are virtually identical, it was not anticipated that one strand would retain two more charges than the other.

The degree of asymmetric charge partitioning increased for an even longer duplex, d19-1, as shown by bar graph summation of the CID data in Figure 2c for the $7-$ through $10-$ charge states. Only in its lowest precursor ion charge state (7-) was the charge distributed symmetrically in the resulting single strand product ions. The most highly charged precursor (10-) yielded the most asymmetric charge partitioning with single strand product ions retaining up to seven or as few as three charges of the 10 available charges. The abundances of the symmetric product ions (5-) are low. The G/A rich strand (ss1) generally retains more charges than the $\mathrm{C} / \mathrm{T}$ rich strand (ss2), but this is not an exclusive rule: either ss1 or ss2 can retain 6 (or 7) charges while the complementary strand only retains 4 (or 3) charges upon CID of the duplex in the 10- charge state. In all cases throughout our study, either strand can end up with the extra charges or deficient in charges, and thus the identity of each strand is not the dominant factor of interest. Examples of the CID mass spectra of d19-1 (charge states 7- through 10-) are shown in Supplemental Figure 3. Based on this initial comparison of duplexes containing from six to 19 base pairs, apparently smaller, lower charged duplexes dis- sociate more symmetrically than larger, more highly charged duplexes.

The degree of asymmetric charge partitioning was examined for four 19 base pair duplexes with varying amounts or positions of A/T base pairs (d19-0, d19-1, d19-2, and d19-3 listed in Table 1). The results are summarized in bar graph form in Figure 3 for all four duplexes in three different charge states $(8-, 9-$, and 10-). CID of d19-3, a duplex which has twice as many A/T base pairs as d19-1 and d19-2, and 20 more A and $\mathrm{T}$ bases as compared to d19-0, displays the most symmetric dissociation. The most asymmetric dissociation occurred for d19-0, the duplex comprised of all G/C and no A/T base pairs, with the $8-$ precursor ion dissociating almost exclusively into single strands that retain 5 and 3 charges (but not 4 and 4), and the 10precursor ion separating into single strands that retain 6 and 4 or 7 and 3 charges (but virtually no 5 and 5). Asymmetric partitioning was also dominant for this duplex in the 9 - charge state, with the formation of

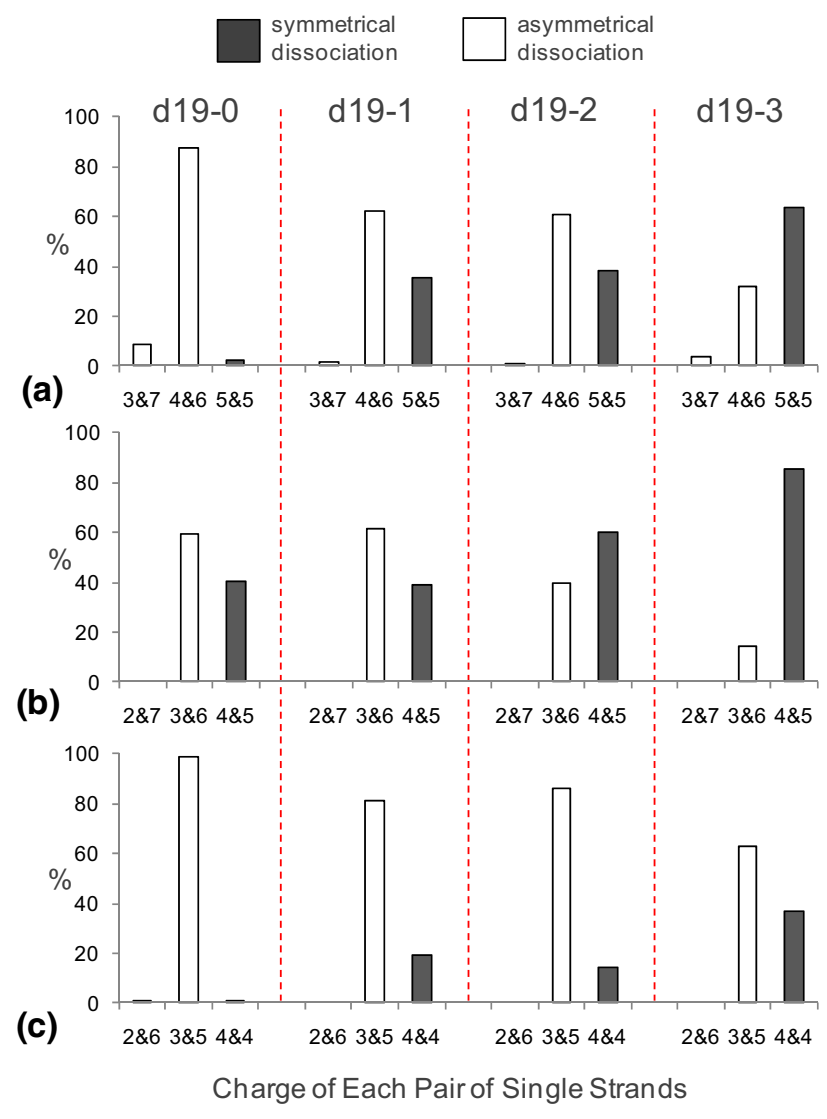

Figure 3. Comparison of symmetrical versus asymmetrical dissociation for 19 mer base pair duplexes with varying A/T base pairs in charge states of (a) 10-, (b) 9-, (c) 8-. Each column represents a different duplex (e.g., d19-0, d19-1, d19-2, and d19-3). The peak areas of the product ions were summed for each pair of single strands and were converted to percentages. The total charge of each pair equaled the precursor charge. The area percentage of product ions involved in charge portioning (asymmetric and symmetric) is plotted against each single strand charge pair. For all duplexes, the CID voltage of (a) 10 - charge state was $48 \mathrm{mV}$, (b) 9- charge state was $51 \mathrm{mV}$, and (c) 8- charge state was $56 \mathrm{mV}$. 
single strands retaining 6 or 3 charges favored over formation of single strands with 5 or 4 charges. The dissociation of duplex d19-0 is particularly interesting because the two constituent strands are nearly identical with one having $10 \mathrm{G}$ and $9 \mathrm{C}$ bases, while the other has $10 \mathrm{C}$ and $9 \mathrm{G}$ bases. The dissociation behavior remains highly asymmetric even upon variation of the CID voltage (which should modulate the energetics of the collisional activation process).

The amount of internal energy deposition has been shown previously to affect the degree of asymmetric charge partitioning observed upon dissociation of multimeric protein complexes [15]. This phenomenon motivated our interest to see if the same was true for DNA complexes. For duplexes in the 10- charge state, the relative portion of asymmetric charge partitioning was determined by summing the abundances of the product ions in the 7-, 3-, 6-, and 4- charge states relative to those in the symmetric 5- charge states for d19-0, $\mathrm{d} 19-1, \mathrm{~d} 19-2$, and d19-3 as a function of the applied CID voltage, as plotted in Supplemental Figure 4. Again, d19-0 exhibits the most asymmetric charge partitioning over the whole range of CID voltages, followed by d19-1 and d19-2, and finally the AT rich d19-3. Examples of the CID mass spectra of d19-3 and d19-0 (charge states 7- through 10-) are shown in Supplemental Figure 5 and Supplemental Figure 6, respectively.

Energy-variable CID was also used to generate the breakdown curves of duplex d14-0 (8- charge state) as shown in Figure 4. The peak areas of the single strand product ions were summed for each type of dissociation behavior (i.e., asymmetric charge partitioning which results in single strands with 6,5 , or 3 charges or symmetric charge partitioning which results in single strands with 4 charges). At lower collision energies, the abundances of single strands arising from symmetric or asymmetric charge partitioning are similar. Conversely, asymmetric dissociation dominates at higher collision energies. This trend in the degree of asymmetric charge partitioning was most notable upon dissociation of duplexes in higher charge states (more asymmetric behavior), as expected based on the previous results that illustrated the impact of the charge state of the precursor ion. Altering the collision voltage caused little or no change in the degree of symmetric versus asymmetric charge partitioning for the duplexes in the lowest charge states which tended to dissociate via more symmetrical charge partitioning.

Given the experimental results described above, the basis for the significant asymmetric charge partitioning upon dissociation of the duplexes may arise from an interplay of conformational effects and intermolecular interactions. Since the dissociation of the duplex d14-2, which consists of two single strands with nearly identical base composition (i.e., one strand containing $8 \mathrm{G}$ and $6 \mathrm{C}$, the other containing $6 \mathrm{G}$ and $8 \mathrm{C}$ ) showed trends in asymmetric charge partitioning similar to the other duplexes in this study, it is unlikely that base composition between the two strands is the deciding factor that affects dissociation behavior (i.e., certain bases facilitate sequestration of more or fewer charges along a strand). Likewise, the dissociation of d19-0, which consists of single strands differing by only one G/C pair (i.e., one strand containing $10 \mathrm{G}$ and $9 \mathrm{C}$, the other containing $10 \mathrm{C}$ and $9 \mathrm{G}$ ), yielded the highest degree of asymmetric charge partitioning in this study, further

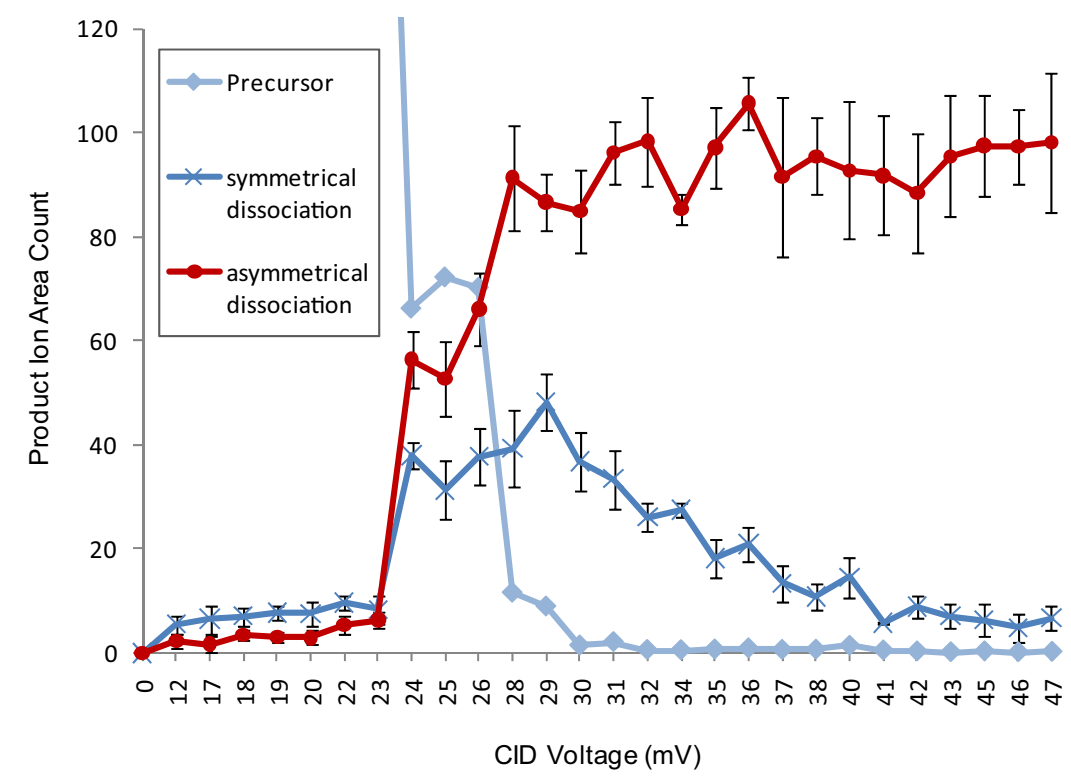

Figure 4. Energy variable CID of d14-0 (8- charge state). The peak areas of the product ions were summed for each type of dissociation charge partitioning (symmetrical versus asymmetrical). The area count of product ions involved in asymmetric dissociation is plotted against CID voltage $(\mathrm{mV}) ; \%$ asymmetric values of $0 \%$ mean the precursor was not significantly dissociated to produce single strands (i.e., $0 \%$ asymmetric does not equal $100 \%$ symmetric dissociation). 
demonstrating that base composition is not dictating the asymmetric versus symmetric charge distribution. The mechanism of asymmetric dissociation of protein complexes has been proposed to be conformation dependent [15]. It has been hypothesized that one subunit of a multimeric complex elongates (e.g., changes its surface area) faster than the others, thus allowing it to become enriched in charge $[9,15]$. Along similar lines, the physical origins of the asymmetric charge partitioning observed for DNA duplexes upon dissociation could also be dependent upon conformation and the rate at which the strands separate relative to proton migration. The four factors investigated in this study that affected the asymmetric charge partitioning of the duplexes were the length and net charge of the duplex (with asymmetric charge partitioning enhanced for longer duplexes in higher charge states), total base composition (i.e., duplexes containing more $\mathrm{G} / \mathrm{C}$ rich base pairs yielded more asymmetric charge partitioning), and internal energy deposition. These factors should collectively influence the initial conformations, the conformational flexibility, proton migration, and the kinetics of disassembly of the duplexes as well as variations in intermolecular interactions and surface areas during disassembly of the duplexes, thus contributing to the observed asymmetric dissociation. The use of molecular modeling to probe conformations and statistical modeling to better understand the constraints that influence the asymmetric versus symmetric behavior would offer more insight for the above hypothesis. Flexibility, conformational changes, and proton transfer would all need to be considered when modeling these duplexes, and the latter factor would make the modeling especially challenging.

\section{Conclusions}

The factors that affect the asymmetric charge partitioning observed upon dissociation of DNA duplexes in the gas phase have been investigated. The degree of asymmetric charge partitioning was shown to increase significantly with duplex size, precursor charge state, G/C base composition, and internal energy deposition. The results parallel many of the previous observations of asymmetric charge partitioning upon dissociation of multimeric protein complexes. Our experimental evidence suggests that the conformations of the duplexes and their propensity for changes in surface area before complete strand separation may dictate the degree of asymmetric charge partitioning, in some ways similar to the mechanisms of protein macromolecular dissociation.

\section{Acknowledgments}

The authors gratefully acknowledge funding from the NIH (R01 GM65956) and the Welch Foundation (F1155).

\section{Appendix A Supplementary Material}

Supplementary material associated with this article may be found in the online version at doi:10.1016/ j.jasms.2010.03.003

\section{References}

1. van den Heuvel, R. H. H.; Heck, A. J. R. Native Protein Mass Spectrometry: From Intact Oligomers to Functional Machineries. Curr. Opin. Chem. Biol. 2004, 8, 519-526.

2. Benesch, J. L. P.; Robinson, C. V. Mass Spectrometry of Macromolecular Assemblies: Preservation and Dissociation. Curr. Opin. Struct. Biol. 2006, 16, 245-251.

3. Felitsyn, N.; Kitova, E. N.; Klassen, J. S. Thermal Decomposition of a Gaseous Multiprotein Complex Studied by Blackbody Infrared Radiative Dissociation. Investigating the Origin of the Asymmetric Dissociation Behavior. Anal. Chem. 2001, 73, 4647-4661.

4. Benesch, J. L. P.; Sobott, F.; Robinson, C. V. Thermal Dissociation of Multimeric Protein Complexes by Using Nanoelectrospray Mass Spectrometry. Anal. Chem. 2003, 75, 2208-2214.

5. Schwartz, B. L.; Bruce, J. E.; Anderson, G. A.; Hofstadler, S. A.; Rockwood, A. L.; Smith, R. D.; Chilkoti, A.; Stayton, P. S. Dissociation of Tetrameric Ions of Noncovalent Streptavidin Complexes Formed by Electrospray Ionization. J. Am. Soc. Mass Spectrom. 1995, 6, 459-465.

6. Versluis, C.; van der Staaij, A.; Stokvis, E.; Heck, A. J. R.; de Craene, B. Metastable Ion Formation and Disparate Charge Separation in the Gas-Phase Dissection of Protein Assemblies Studied by Orthogonal Time-of-Flight Mass Spectrometry. J. Am. Soc. Mass Spectrom. 2001, 12, 329-336.

7. Zhang, Z.; Krutchinsky, A.; Endicott, S.; Realini, C.; Rechsteiner, M.; Standing, K. G. Proteasome Activator 11S REG or PA28: Recombinant REG $\alpha /$ REG $\beta$ Hetero-oligomers Are Heptamers. Biochemistry 1999, 38, 5651-5658.

8. Rostom, A. A.; Sunde, M.; Richardson, S. J.; Schreiber, G.; Jarvis, S.; Bateman, R.; Dobson, C. M.; Robinson, C. V. Dissection of Multi-Protein Complexes Using Mass Spectrometry: Subunit Interactions in Transthyretin and Retinol-Binding Protein Complexes. Proteins: Struct. Funct. Genet. 1998, 33, 3-11.

9. Light-Wahl, K. J.; Schwartz, B. L.; Smith, R. D. Observation of the Noncovalent Quaternary Associations of Proteins by Electrospray Ionization Mass Spectrometry. J. Am. Chem. Soc. 1994, 116, 5271-5278.

10. Fitzgerald, M. C.; Chernushevich, I.; Standing, k. G.; Whitman, C. P.; Kent, S. B. H. Probing the Oligomeric Structure of an Enzyme by Electrospray Ionization Time-of-Flight Mass Spectrometry. Proc. Natl. Acad. Sci. U.S.A. 1996, 93, 6851-6856.

11. Rostom, A. A.; Fucini, P.; Benjamin, D. R.; Juenemann, R.; Nierhaus, K. H.; Hartl, F. U.; Dobson, C. M.; Robinson, C. V. Detection and Selective Dissociation of Intact Ribosomes in a Mass Spectrometer. Proc. Natl. Acad. Sci. U.S.A. 2000, 97, 5185-5190.

12. Fandrich, M.; Tito, M. A.; Leroux, M. R.; Rostom, A. A.; Hartl, F. U.; Dobson, C. M.; Robinson, C. V. Observation of the Noncovalent Assembly and Disassembly Pathways of the Chaperone Complex MtGimC by Mass Spectrometry. Proc. Natl. Acad. Sci. U.S.A. 2000, 97, 14151-14155.

13. Aquilina, J. A.; Benesch, J. L. P.; Bateman, O. A.; Slingsby, C.; Robinson, C. V. Polydispersity of a Mammalian Chaperone: Mass Spectrometry Reveals the Population of Oligomers in $\alpha$ B-crystallin. Proc. Natl. Acad. Sci. U.S.A. 2003, 100, 10611-10616.

14. Benesch, J. L. P.; Aquilina, J. A.; Ruotolo, B. T.; Sobott, F.; Robinson, C. V. Tandem Mass Spectrometry Reveals the Quaternary Organization of Macromolecular Assemblies. Chem. Biol. 2006, 13, 597-605.

15. Jurchen, J. C.; Williams, E. R. Origin of Asymmetric Charge Partitioning in the Dissociation of Gas-Phase Protein Homodimers. J. Am. Chem. Soc. 2003, 125, 2817-2826.

16. Jurchen, J. C.; Garcia, D. E.; Williams, E. R. Further Studies on the Origins of Asymmetric Charge Partitioning in Protein Homodimers. J. Am. Soc. Mass. Spectrom. 2004, 15, 1408-1415.

17. Wanasundara, S. N.; Thachuk, M. Theoretical Investigations of the Dissociation of Charged Protein Complexes in the Gas Phase. J. Am. Soc. Mass Spectrom. 2007, 18, 2242-2253.

18. Csiszar, S.; Thachuk, M. Using Ellipsoids to Model Charge Distributions in Gas Phase Protein Complex Ion Dissociation. Can. J. Chem. 2004, 82, $1736-1744$

19. Wanasundara, S. N.; Thachuk, M. Free Energy Barrier Estimation for the Dissociation of Charged Protein Complexes in the Gas Phase. J. Phys. Chem. A 2009, 113, 3814-3821.

20. Jones, C. M.; Beardsley, R. L.; Galhena, A. S.; Dagan, S.; Cheng, G.; Wysocki, V. H. Symmetrical Gas-Phase Dissociation of Noncovalent Protein Complexes via Surface Collisions. J. Am. Chem. Soc. 2006, 128, 15044-15045.

21. Beardsley, R. L.; Jones, C. M.; Galhena, A. S.; Wysocki, V. H. Noncovalent Protein Tetramers and Pentamers with " $n$ " Charges 
Yield Monomers with n/4 and n/5 Charges. Anal. Chem. 2009, 81, 1347-1356.

22. Schnier, P. D.; Klassen, J. S.; Strittmatter, E. F.; Williams, E. R. Activation Energies for Dissociation of Double Strand Oligonucleotide Anions: Evidence for Watson-Crick Base Pairing In Vacuo. J. Am. Chem. Soc. 1998, 120, 9605-9613.

23. Gabelica, V.; De Pauw, E. Collision-Induced Dissociation of 16-mer DNA Duplexes with Various Sequences: Evidence for Conservation of the Double Helix Conformation in the Gas Phase. Int. J. Mass Spectrom. 2002, 219, 151-159.

24. Gabelica, V.; De Pauw, E. Comparison Between Solution-Phase Stability and Gas-Phase Kinetic Stability of Oligodeoxynucleotide Duplexes. J. Mass Spectrom. 2001, 36, 397-402.

25. Rueda, M.; Kalko, S. G.; Luque, F. J.; Orozco, M. The Structure and Dynamics of DNA in the Gas Phase. J. Am. Chem. Soc. 2003, 125, 8007-8014.
26. Danell, A. S.; Parks, J. H. Fraying and Electron Autodetachment Dynamics of Trapped Gas-Phase Oligonucleotides. J. Am. Soc. Mass Spectrom. 2003, 14, 1330-1339.

27. Danell, A. S.; Parks, J. H. FRET Measurements of Trapped Oligonucleotide Duplexes. Int. J. Mass Spectrom. 2003, 229, 35-45.

28. Gabelica, V.; De Pauw, E. Comparison of the Collision-Induced dissociation of duplex DNA at different collision regimes: Evidence for a Multistep Dissociation Mechanism. J. Am. Soc. Mass Spectrom. 2002, 13, 91-98.

29. Gidden, J.; Ferzoco, A.; Baker, E. S.; Bowers, M. T. Duplex Formation and the Onset of Helicity in Poly d(CG)n Oligonucleotides in Solvent-Free Environment. I. Am. Chem. Soc. 2004, 126, 15132-15140.

30. Gidden, J.; Baker, E. S.; Ferzoco, A.; Bowers, M. T. Structural Motifs of DNA Complexes in the Gas Phase. Int. J. Mass Spectrom. 2005, 240, 183-193.

31. Baker, E. S.; Bowers, M. T. B. -DNA Helix Stability in a Solvent-Free Environment. J. Am. Soc. Mass Spectrom. 2007, 18, 1188-1195. 